\title{
Investigation of inter-ELM pedestal profiles in ASDEX Upgrade
}

\author{
E. Wolfrum, A. Burckhart, R. Fischer, N. Hicks, C. Konz, B. Kurzan, B. \\ Langer, T. Pütterich , H. Zohm and the ASDEX Upgrade Team \\ Max Planck Institut für Plasmaphysik, EURATOM Association, Garching, \\ Germany \\ E-mail: e.wolfrum@ipp.mpg.de
}

\begin{abstract}
Integrated data analysis and equilibrium reconstruction on a millisecond time base are used to gain edge profiles with high spatial and temporal resolution. The measured radial electric field profiles for a series of discharges with different gas fuelling levels show their minimum at the position of maximal ratio of pressure gradient and density $(\nabla \mathrm{p} / \mathrm{n})$. The analysis of electron density and temperature profiles in between ELMs reveals a characteristic sequence of phases, starting with a fast recovery phase, a quiet pressure build up phase and a strongly fluctuating phase before the next ELM breaks out. These phases are described in terms of profile development, the behaviour of maximal gradients as well as their positions.
\end{abstract}

PACS: 52.55.Fa, 52.55.Fi

\section{Introduction}

For ITER the H-mode is the foreseen operating scenario. In present day machines most $\mathrm{H}$-modes are characterised by the formation of a strong edge transport barrier (ETB), which is also called pedestal, and the occurrence of edge localised modes (ELMs). Large efforts are put into characterisation of the pedestal and its scaling $[1,2,3,4,5]$ as well as in understanding the physics of ELMs [6], but the predictions for ITER are still unclear. In order to improve the understanding of the formation of the pedestal, measurements with high spatial and temporal resolution can contribute to the refinement of models.

The aim of this work is to show the current capabilities of pedestal measurements at ASDEX Upgrade and demonstrate on a set of data the latest experimental findings. During the last two years innovative data evaluation techniques using Bayesian probability theory in an integrated approach of data analysis were set up at ASDEX Upgrade [7]. In that way the electron density $\left(n_{e}\right)$ profiles [8] and the profiles of the radial electric field $E_{r}[9]$ are determined including proper 
uncertainties. Together with high resolution electron temperature $\left(T_{e}\right)$ [10], ion temperature $\left(T_{i}\right)$ and toroidal velocity $\left(\mathrm{v}_{\text {tor }}\right.$ ) profiles [11] the pedestal is well described.

The paper is divided into 3 sections. First we describe a series of discharges in H-mode with same heating powers, where the gas fuelling level was changed from 0 to $10^{22} \mathrm{~s}^{-1}$. In the next section, we present inter-ELM edge profiles for these discharges. In the last section we present the development of $\mathrm{n}_{\mathrm{e}}$ and $\mathrm{T}_{\mathrm{e}}$ profiles in between ELMs with high temporal resolution.

\section{Series of discharges with different gas fuelling levels}

The analysis of profiles in the edge transport barrier (ETB) is carried out for a series of discharges, with a plasma current of $1 \mathrm{MA}$ at $-2.5 \mathrm{~T}$, heated with $7.5 \mathrm{MW}$ of neutral beam heating (NBI) and 1.3 MW of central ECRH heating. The discharges have a lower x-point, a value of $\mathrm{q}_{95}$ around 4.7, with an upper triangularity of $0.16,0.12,0.123,0.18$ and a lower triangularity of $0.44,0.415,0.41$ and 0.4 for discharges 23226, 23219, 23221 and 23225, respectively.

The amount of $\mathrm{D}_{2}$ fuelling was varied from 0 (\#23226) to $4 \cdot 10^{21} \mathrm{~s}^{-1}$ (\#23219), $5.8 \cdot 10^{21} \mathrm{~s}^{-1}$ (\#23221) and $9 \cdot 10^{21} \mathrm{~s}^{-1}$ (\#23225). All fuelled discharges have the same confinement $\left(\mathrm{H}_{98} \sim 0.93\right)$, in the one without fuelling the confinement is $10 \%$ higher $\left(\mathrm{H}_{98}=1.02\right)$. Discharges \#23219 and \#23221 include radial sweeps to improve the spatial resolution of edge diagnostics. The profiles presented in section 3 are inter-ELM profiles, where the profiles are ELM synchronized and averaged in the time range of $-3.5 \mathrm{~ms}$ to $-1 \mathrm{~ms}$ before an ELM. For the profile data with high temporal resolution presented in section 4 the radial sweep is excluded, as the ELM behaviour is slightly modified during such radial sweeps, which might be due to the change of neutral particle recycling with the distance between plasma and wall or to the varying magnetic field ripple amplitude.

Figure 1 shows time traces of the four discharges: the line integrated density (core DCN channel, top frame) varies according to the fuelling level (second frame). The ELM frequency (third frame) is lowest with $80 \pm 17 \mathrm{~Hz}$ with the highest fuelling level, and is $125 \pm 22(24) \mathrm{Hz}$ for the medium fuelled discharges. The zero fuelling level discharge exhibits an ELM frequency of 104 $\pm 36 \mathrm{~Hz}$ and shows periods with missing ELMs, reflected in the large standard deviation of the ELM frequency. The ELM signature is shown with the WI intensity measured in the divertor, because the signal is much sharper, i.e. consists of a single maximum per ELM, and therefore better suited to determine the ELM onset time than the $\mathrm{H}_{\alpha}$ signal in a full tungsten divertor. Although the magnetic measurements have a better temporal resolution, the WI signal is used for automatic ELM time determination because of its unambiguous shape. Usually, the ELM frequency of type-I ELMs increases with higher gas fuelling. In this series of discharges the ELM frequency of the discharge with the largest fuelling rate is the lowest. An attempt for an explanation will be given in section 4 . 

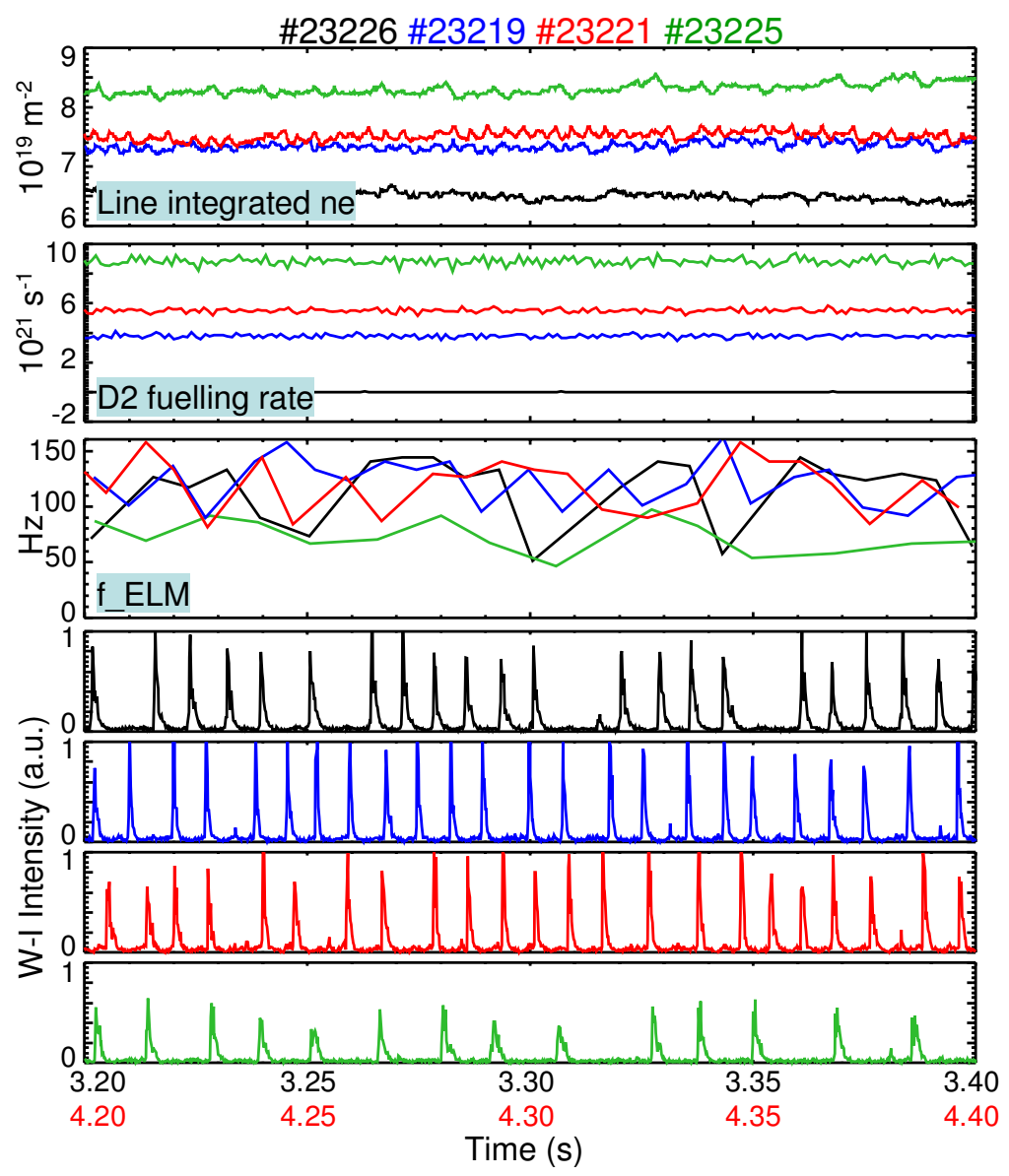

Figure 1: Timetraces of the line integrated electron density, the $\mathrm{D}_{2}$ fuelling rate, the ELM frequency and the WI intensity as measured in the divertor to show the ELM signature for discharges \#23226 (black), \#23219 (blue), \#23221 (red) and \#23225 (green). The lower (red) time axis is valid for \#23221, the upper (black) one for all other discharges.

\section{Inter-ELM edge profiles}

At ASDEX Upgrade $T_{e}$ edge profiles are measured with electron cyclotron emission (ECE) with a sampling rate of $31 \mathrm{kHz}$ and a spatial resolution of $1 \mathrm{~cm} / 6 /$. ECE data presented here are not affected by ECE shine-through, which perturb the data in regions of insufficient optical depth. $n_{e}$ edge profiles are determined by the lithium beam diagnostic (LIB) with $20 \mathrm{kHz}$. Integrated data analysis, combining the DCN data (laser interferometry) with the LIB data /4/ yield $\mathrm{n}_{\mathrm{e}}$ profiles with excellent spatial resolution of $5 \mathrm{~mm}$ in the ETB and reduced uncertainties at the pedestal top due to the additional information from the DCN channels. Edge ion temperature profiles as well as toroidal velocity profiles stem from charge exchange recombination spectroscopy (CXRS) diagnostics /7/ with a temporal resolution of $1.9 \mathrm{~ms}$ and a spatial resolution of between 3 and 8 $\mathrm{mm}$, depending on the line of sight and on the density gradient. 
Radial electric field profiles are evaluated with a Bayes algorithm from line integrated HeII emission profiles $/ 5 /$. The radial resolution determined by the optical system is $5 \mathrm{~mm}$. Most other sources of uncertainty are taken into account by the Bayes algorithm, except the uncertainty in the equilibrium. The temporal resolution of $4 \mathrm{~ms}$ is determined by the exposure time of the frame transfer CCD. For the analysis, all frames which contain an ELM are omitted, so that only frames in between ELMs are used for the reconstruction of $E_{r}$ profiles. The profiles are mapped to the magnetic midplane and $n_{e}$ and $T_{e}$ data from the Thomson scattering diagnostic are used to verify the relative alignment of the other diagnostics $[12,13]$. For $1 \mathrm{MA},-2.5 \mathrm{~T}$ discharges the pedestal widths vary in the range of $1.7 \pm 0.5 \mathrm{~cm}$ for $\mathrm{n}_{\mathrm{e}}$ and $2 \pm 0.5 \mathrm{~cm}$ for $\mathrm{T}_{\mathrm{e}}$ in ASDEX Upgrade, so that the spatial resolution of all diagnostics is sufficient to resolve the edge transport barrier.

Figure 2 shows inter-ELM profiles of the discharge with no gas fuelling (\#23226), one with medium gas fuelling (\#23221) and one with high fuelling (\#23225) versus normalized poloidal radius $\rho_{\text {pol }}$, with $\rho_{\text {pol }}=\operatorname{sqrt}\left[\left(\psi-\psi_{\mathrm{a}}\right) /\left(\psi_{\mathrm{s}}-\psi_{\mathrm{a}}\right)\right]$, where $\psi$ is the poloidal flux, a refers to the magnetic axis and $\mathrm{s}$ to the separatrix. The $\mathrm{n}_{\mathrm{e}}$ and $\mathrm{T}_{\mathrm{e}}$ data are fitted with a modified tanh function [14]. Only discharge \#23221 has a radial sweep which can easily be seen in the better resolution of the $T_{i}$ and $\mathrm{v}_{\text {tor }}$ profiles. Ion temperatures exhibit the same profile shape as electron temperatures, with a slightly higher $(\sim 10 \%)$ pedestal top value. The minimum in the toroidal velocity profile $/ 7 /$ is visible in all three discharges, but the scatter of the data is such that no distinct difference can be seen due to the changed gas fuelling. In contrast, the minimum of the radial electric field is deeper with lower density, which would be expected if $E_{\mathrm{r}}$ is proportional to $\nabla \mathrm{p}_{\mathrm{i}} / \mathrm{n}_{\mathrm{i}}$. The absolute values of the $\mathrm{E}_{\mathrm{r}}$ minimum presented here are considerably higher than comparable ELM resolved measurements from Doppler reflectometry, which exhibit minima in the range of $-30 \mathrm{kV} / \mathrm{m}$ [15]. The position of the $\mathrm{E}_{\mathrm{r}}$ minimum coincides with the position of maximal $\nabla \mathrm{p}_{\mathrm{i}} / \mathrm{n}_{\mathrm{i}}$, assuming that $\mathrm{n}_{\mathrm{i}}$ is proportional to $\mathrm{n}_{\mathrm{e}}$.

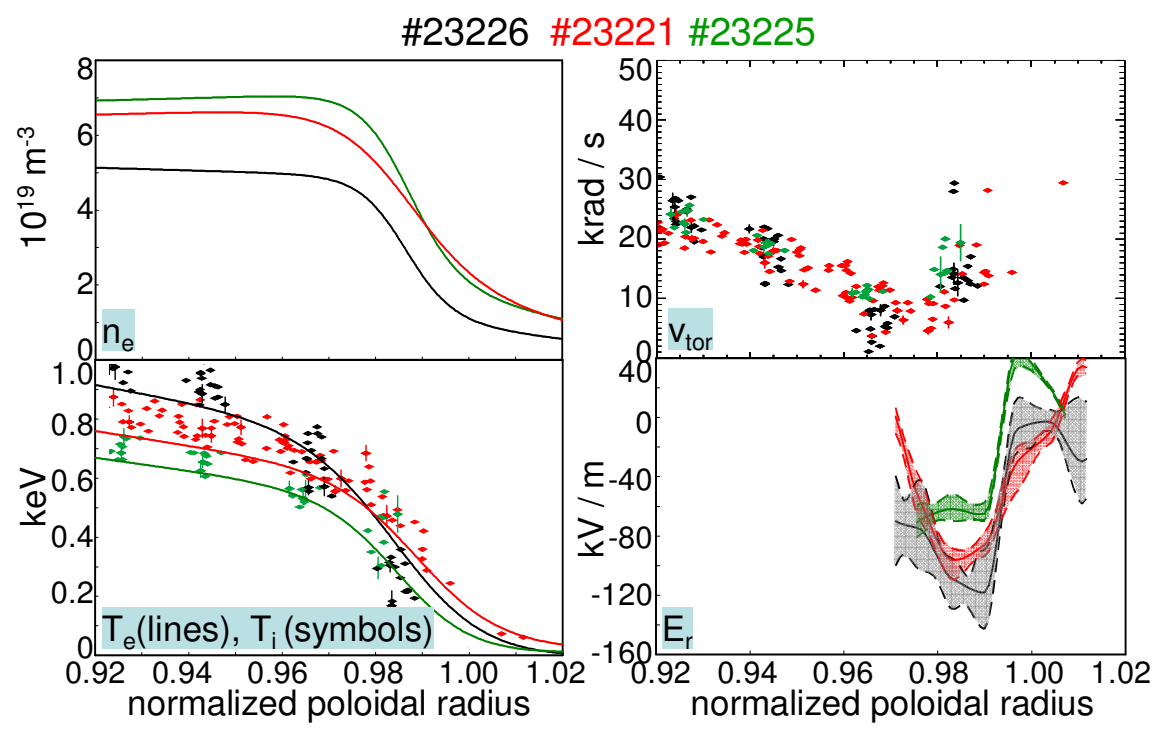

Figure 2: Inter-ELM profiles of $\mathrm{n}_{\mathrm{e}}, \mathrm{T}_{\mathrm{e}}$ and $\mathrm{T}_{\mathrm{i}}, \mathrm{v}_{\text {tor }}$ and $\mathrm{E}_{\mathrm{r}}$ for \#23226 (black, no gas fuelling), \#23221 (red, 5.8·10 $21 \mathrm{~s}^{-1}$ ) and \#23225 (green, $9 \cdot 10^{21} \mathrm{~s}^{-1}$ ). 


\section{Profiles in between ELMs with high temporal resolution}

In order to get accurate information from ECE data with respect to the temporal behaviour of the ETB region, it is mandatory to provide a magnetic equilibrium with high temporal resolution. The data presented here use CLISTE equilibria [16] with a temporal resolution of $1 \mathrm{~ms}$. Also the integrated data analysis (IDA) algorithm must use the fast equilibrium to combine LIB and DCN data. The most influential effect of the equilibrium on the profiles is due to the fast movement of the separatrix position with or after an ELM. As can be seen in figure 3 the outer separatrix position moves by $8-10 \mathrm{~mm}$ in every ELM cycle. With ETB widths of $12-20 \mathrm{~mm}$ this means that e.g. an ECE channel can cover the top and the bottom of the pedestal in the same ELM cycle.

In figure 3, different plasma properties are shown over a time interval including four ELMs. The magnetic pick-up coil signal is shown as ELM reference. The outer separatrix position is calculated with a temporal resolution of $1 \mathrm{~ms}$, as mentioned above. While the outer separatrix moves inwards during the ELM, at the high field side the separatrix moves towards larger R. This means that on both sides, the high and low field side, the separatrix moves towards the plasma center. At the same time the X-point is pulled about $8 \mathrm{~mm}$ downwards, and the plasma volume shrinks.

The evolution of profiles can be seen in the time traces of $\mathrm{T}_{\mathrm{e}}, \mathrm{n}_{\mathrm{e}}$ and $\mathrm{p}_{\mathrm{e}}$ at fixed poloidal radius of 0.99 (red) and 0.98 (black) in figure 3. The time points indicated by dashed lines are the time points of the ELM crashes as seen in the magnetic pick up coil (top trace).

When comparing the electron temperature at a fixed poloidal radius to the outer separatrix position, it can be noted that the equilibrium reconstruction influences the temperature evolution. As an example, this can be observed after the second ELM crash shown in figure 3. Here it seems that following the fast rise phase $\mathrm{T}_{\mathrm{e}}$ continues to increase slowly, but then drops again. This behavior is due to the probably inaccurate equilibrium reconstruction, as can be seen from the correlation of such a drop in $T_{e}$ with the position of the outer separatrix. Note, that the equilibria are not calculated taking kinetic edge pressure profiles into account self consistently. If one assumes an equilibrium adjustment during an ELM, then a continuous growth of the plasma volume, and finally a constant equilibrium until the next ELM, the electron temperature behaviour can be described in three phases, as observed in the third ELM of figure 3.

Typically, the $\mathrm{T}_{\mathrm{e}}$ values in the ETB region drop during an ELM and take about $2 \mathrm{~ms}$ to recover to a steady value. After further 2-3 ms there is a transition to a third phase: $T_{e}$ rises continuously with large amplitudes in the $T_{e}$ values, probably indicating the occurrence of fluctuations or filaments.

The $n_{e}$ signal is generally noisier than the $T_{e}$ signals, but also here a first recovery phase, which takes around $4 \mathrm{~ms}$ can be observed. Thereafter, depending on the gas fuelling level a transient increase in $n_{e}$ can be seen, which is assumed to be due to recycling. After about $6 \mathrm{~ms}_{\mathrm{e}}$ reaches a constant, but strongly fluctuating level.

In the strongly fluctuating phase, most of the large drops (spikes towards lower temperatures) visible in the $T_{e}$ signal can be correlated with positive spikes in $n_{e}$. These features seem to be turbulent structures or filaments moving in or originating in the pedestal. Note that both diagnostics are positioned in the same sector of ASDEX Upgrade, at the same z-position but toroidally separated by about $30 \mathrm{~cm}$, so that structures with enough poloidal extent (minimum 5$10 \mathrm{~cm}$ depending on field line angle, which depends on the bootstrap current in this region) can pass both diagnostics along field lines. The analysis of data from Thomson scattering [17] show that holes appear on the inside of the ETB and blobs are observed at the outside, while the pdfs (probability distribution functions) are symmetric at the position close to the middle of the ETB region. 


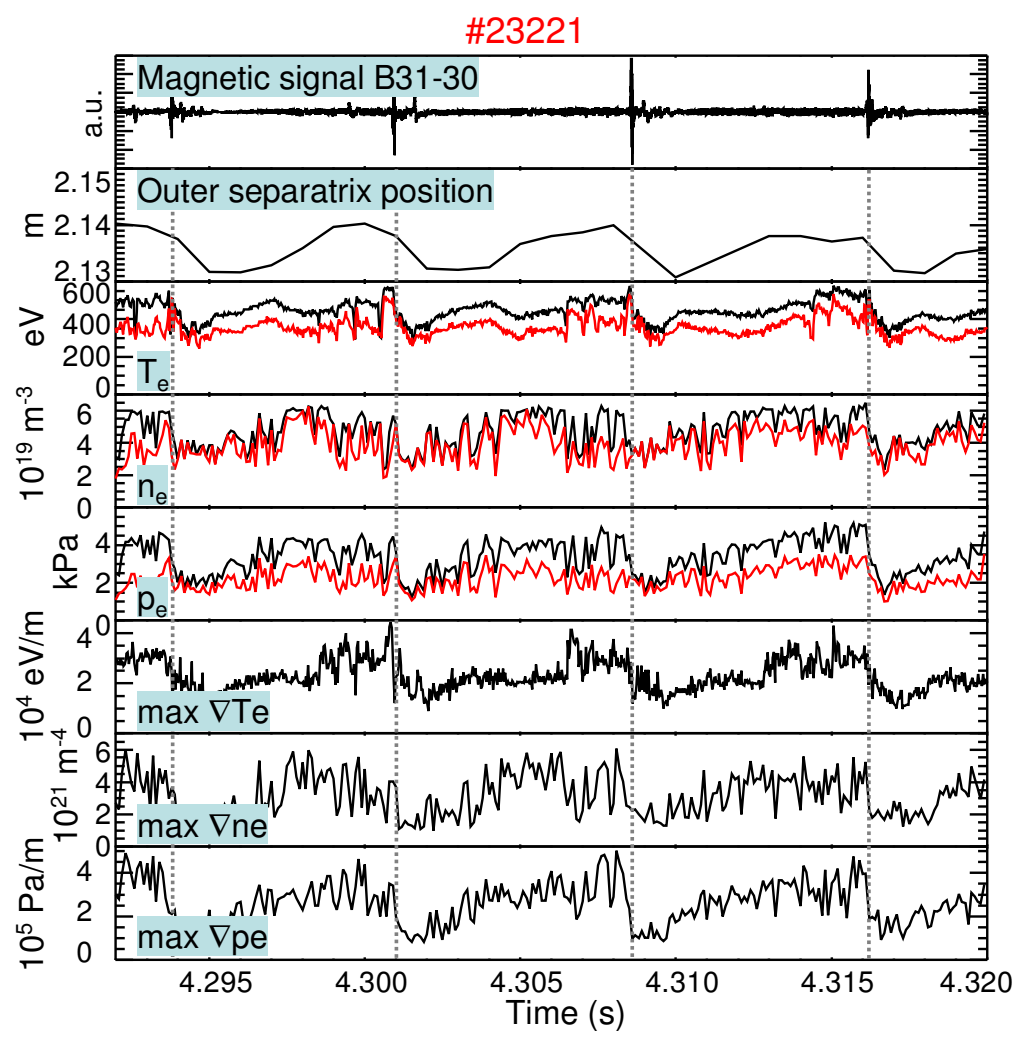

Figure 3: Four ELM cycles of discharge \#23221: Magnetic signal, outer separatrix position, $\mathrm{T}_{\mathrm{e}}, \mathrm{n}_{\mathrm{e}}$ and $\mathrm{p}_{\mathrm{e}}$ at $\rho_{\mathrm{pol}}=0.98$ (black) and $\rho_{\text {pol }}=0.99$ (red), maximum $\mathrm{T}_{\mathrm{e}}, \mathrm{n}_{\mathrm{e}}$ and $\mathrm{p}_{\mathrm{e}}$ gradients.

The behaviour of the maximal temperature gradient shows the same evolution as the $T_{e}$ values in the pedestal region, but it is not subject to possible inaccuracies in the equilibrium calculation. During the second, "smooth" phase, the position of the maximal temperature gradient lies around $\rho_{\text {pol }}=0.985$. When entering the fluctuating phase, it moves to a position between $\rho_{\text {pol }}=0.99$ and 1.00. The accuracy with which this position can be determined is limited by the spatial resolution of the ECE diagnostic whose measured data points lie about $1 \mathrm{~cm}$ apart.

The maximal density gradient shows the same behavior as the $\mathrm{n}_{\mathrm{e}}$ value itself. Its position tends to move slightly inwards during the recovery from the ELM crash, from $\rho_{\text {pol }}=1.00$ to $\rho_{\text {pol }}=0.99$.

The maximal electron pressure gradient shows a first rapid recovery phase about 1-2ms after the ELM crash, and then a long steady increase until the next ELM.

For all four discharges, the data from $\mathrm{T}_{\mathrm{e}}, \mathrm{n}_{\mathrm{e}}$ and $\mathrm{p}_{\mathrm{e}}$ were drawn on a scatter plot relative to the ELM onset time. Figure 4 shows the recovery of the maximal $\mathrm{T}_{\mathrm{e}}$ gradient after the ELM crash for discharge 23221. In order to compare the discharges and study the influence of gas fuelling on edge profiles, the mean value and standard deviation were calculated for every $0.1 \mathrm{~ms}$ interval (red curve). Note that the error bars displayed on figure 4 and 5 portray the standard deviation of the scatter and do not include the uncertainties of the measurements.

Figure 5 shows the ELM-synchronized behaviour of the maximal gradients (left) and values at $\rho_{\text {pol }}=0.99$ (right) of $\mathrm{T}_{\mathrm{e}}, \mathrm{n}_{\mathrm{e}}$ and $\mathrm{p}_{\mathrm{e}}$. 
The maximal $\mathrm{T}_{\mathrm{e}}$ gradient shows the three phases described earlier: fast recovery phase $(2 \mathrm{~ms})$, smooth constant phase $(3 \mathrm{~ms})$ and strongly fluctuating phase until the next ELM occurs.

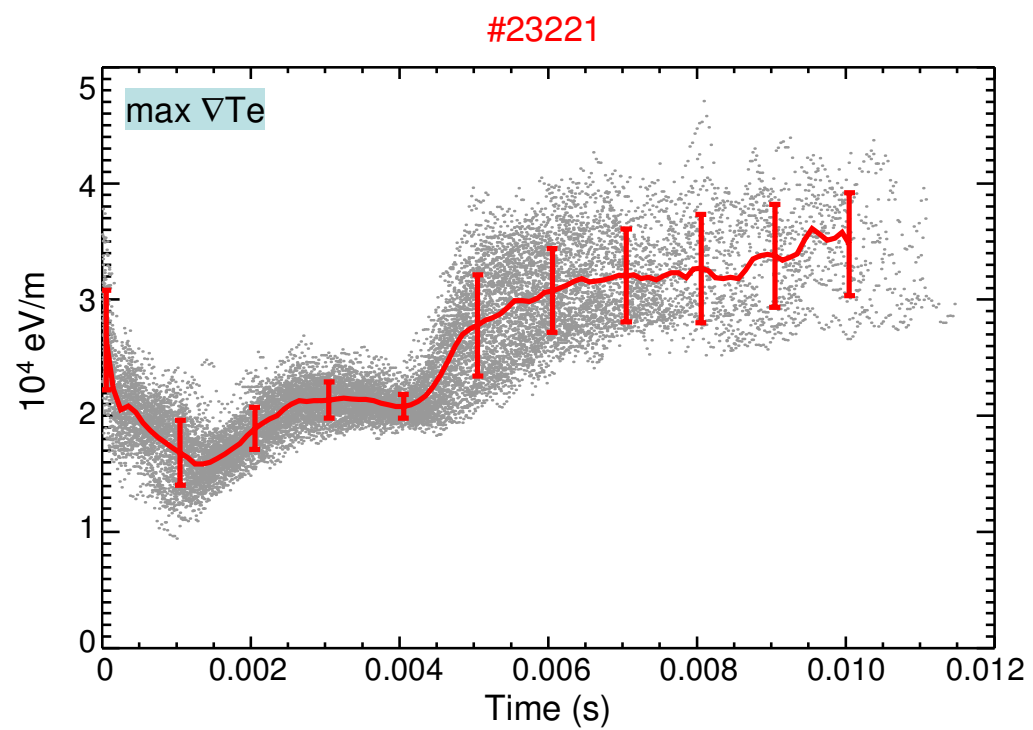

Figure 4: Maximal $\mathrm{T}_{\mathrm{e}}$ gradient values plotted relative to the ELM onset time (grey dots). $\mathrm{t}=0$ corresponds to ELM start time. Red curve: mean value of $0.1 \mathrm{~ms}$ intervals, error bars: standard deviation.

As can be seen on discharge 23225, with high gas fuelling it becomes difficult to distinguish the different phases. Furthermore, the temperature gradient does not get to the high values it reaches without gas. In the last phase, shortly before the next ELM, the temperature values at $\rho_{\mathrm{pol}}=0.99$ for the discharges without and with moderate gas fuelling are practically identical, whereas the massive fuelling on discharge 23225 causes a $30 \%-40 \%$ lower electron temperature.

The scatter plots of $n_{e}$ exhibit a very strong scatter, which is represented by the large standard deviation of the mean value. All trends lie within these errors. When looking at $n_{e}$, the shot with no gas fuelling has a qualitatively different time evolution than the discharges with gas fuelling. With more gas, the density gradient recovers more slowly, but it still reaches the same final value of about $4 \cdot 10^{21} \mathrm{~m}^{-4}$ as with lower gas fuelling. In the case without fuelling, the overall electron density is too low for the gradient to reach this value and $n_{e}$ at $\rho_{\text {pol }}=0.99$ does not show the distinct maximum between $3 \mathrm{~ms}$ and $6 \mathrm{~ms}$ after the ELM. This peak is suspected to result from recycling of neutral particles which enter the plasma after the ELM crash. Temporal developments of $n_{e}$ profiles for single ELMs are described in [18].

The maximum $\nabla \mathrm{p}_{\mathrm{e}}$ value reaches the same pre-ELM level for all four discharges. With high gas fuelling, $\nabla \mathrm{p}_{\mathrm{e}}$ takes longer to recover from the ELM, but its final value is similar to the other discharges. The $p_{e}$ values at $\rho_{\text {pol }}=0.99$ decrease with gas fuelling, except that the discharge without fuelling is roughly at the same level as the one with the highest fuelling. This is also reflected in the ELM frequency, where in the case with the highest fuelling the ELM frequency is lowest $(\sim 80 \mathrm{~Hz})$ and in the case with no fuelling there are missing ELMs. 

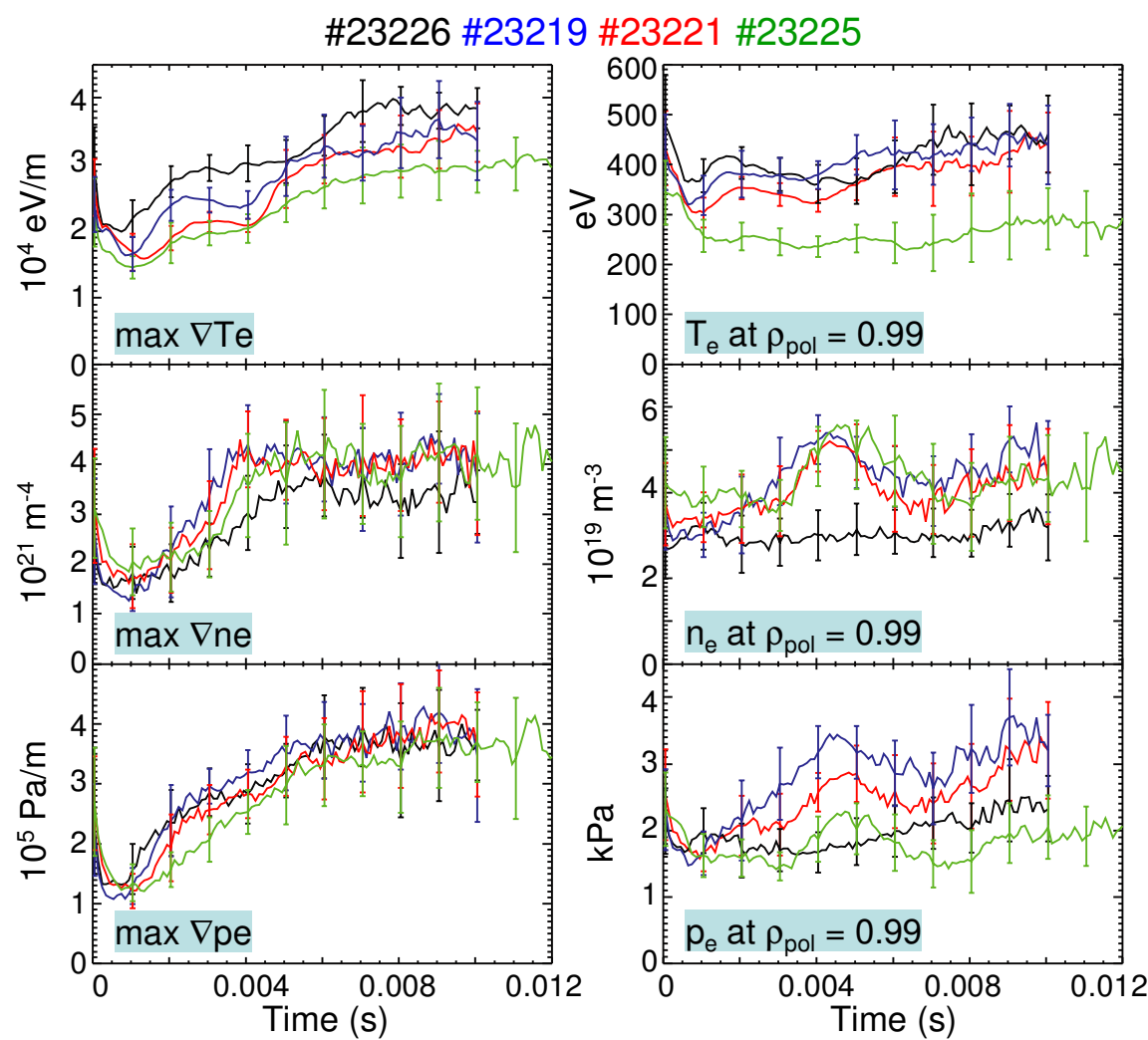

Figure 5: Comparison of ELM synchronized data for discharges with different gas fuelling levels (\#23226 black, \#23219 blue, \#23221 red, \#23225 green, fuelling increasing from 0 to $9 \cdot 10^{21} \mathrm{~s}^{-1}$ ). Left: maximal values of $\nabla \mathrm{T}_{\mathrm{e}}, \nabla \mathrm{n}_{\mathrm{e}}$ and $\nabla \mathrm{p}_{\mathrm{e}}$, right: $\mathrm{T}_{\mathrm{e}}, \mathrm{n}_{\mathrm{e}}$ and $\mathrm{p}_{\mathrm{e}}$ at $\rho_{\mathrm{pol}}=0.99$.

\section{Conclusions}

The data presented here cover ELM resolved edge profiles for four discharges with different gas fuelling levels but same heating power. The evolution of electron density and temperature profiles show distinctly different and uncorrelated behaviours. The density gradient recovers on a time scale which is independent of the $T_{e}$ gradient behaviour. The recovery of the maximal $n_{e}$ gradient is slower for higher fuelling and the values reach an upper limit, except for the discharge with no gas fuelling.

From the $\mathrm{T}_{\mathrm{e}}$ data it is obvious that two different transport mechanisms for electrons are present: (i) one with small scales, which cannot be resolved by our diagnostics, dominating the initial pressure build up phase, and (ii) one with large scales, causing the large fluctuating signal in $T_{e}$ and $n_{e}$ in the phase where the pressure gradient has reached a plateau level. Interesting in this context is a comparison of various electron heat transport models with data from Thomson scattering, which show that electron heat diffusivity is due to small turbulent structures [19] and that electron heat transport takes place on scales down to the collisionless skin depth. 
The electron pressure gradient seems to be the governing quantity. For all four cases the pressure gradient has the same upper limit, which is reached well before the ELM breaks out. This is in agreement with the assumption that ballooning instabilities limit the pressure gradient, but do not trigger the ELM. As the build up of bootstrap current takes some time, it might be that peeling modes develop which lead to the ELM break out [20]. This is part of ongoing investigations.

The radial electric field behaves as expected from neoclassical theory, namely that its minimum is positioned where $\nabla p_{i} / n_{i}$ is maximal. Given that $\nabla p_{i}$ reaches the same pre-ELM level in all discharges $E_{r}$ scales with $n_{i}^{-1}$, if $n_{i}$ is assumed to be equal to $n_{e}$ and taken at the pedestal top.

The minimum in the toroidal velocity profile does not show a dependence on the change in gas fuelling within the error bars of the measurements.

\section{References}

1 A. Hubbard, Plasma Phys. Controlled Fusion 42 (2000) A15

2 A. Kirk, Phys. Controlled Fusion 50 (2004) A187

3 C. F. Maggi et al., Nucl.Fusion 47 (2007) 535

4 A.W. Leonard, J.Physics: Conf. Series 123 (2008) 012001

$5 \quad$ P.B. Snyder et al., Nucl.Fusion 49 (2009) 085035

6 N. Oyama, Conf. Series 123 (2008) 012002

7 R. Fischer et al., Rev. Sci. Instrum. 75 (2004) 4237

8 R. Fischer et al., Plasma Phys. Controlled Fusion 50 (2008) 085009

9 E. Wolfrum et al., $35^{\text {th }}$ EPS Conf. on Plasma Physics (Hersonissos, Greece, 2008) 2.002

10 N.K. Hicks et al., to be published in Proc. $15^{\text {th }}$ Joint Workshop ECE and ECRH (2008)

11 T. Pütterich et al., PRL 102 (2009) 025001

12 J. Neuhauser et al, Plasma Phys. Controlled Fusion 44 (2002) 855

13 A. Kallenbach et al., J.Nucl.Materials 337-339 (2005) 381

14 R.J. Groebner et al., Nucl. Fusion 41 (2001) 1789

15 G.D. Conway et al., Proc. $16^{\text {th }}$ European Fusion Physics Workshop (Cork, Ireland, 2008)

16 P. McCarthy et al., $30^{\text {th }}$ EPS Conf. on Plasma Physics (St.Petersburg, Russia, 2003) P1.64

17 B. Kurzan et al., Plasma Phys. Controlled Fusion 51 (2009) 065009

18 R. Fischer et al., $36^{\text {th }}$ EPS Conf. on Plasma Physics (Sofia, Bulgaria, 2009) P1.159

19 B. Kurzan et al., $36^{\text {th }}$ EPS Conf. on Plasma Physics (Sofia, Bulgaria, 2009) P1.153

20 H. Zohm, Plasma Phys. Controlled Fusion 38 (1996) 105 